\title{
A CONTROL SYSTEM FOR THE DUKE BOOSTER SYNCHROTRON*
}

\author{
S. M. Hartman ${ }^{\dagger}$, S. F. Mikhailov, Y. K. Wu, \\ FEL Laboratory, Duke University, Durham, North Carolina 27708-0319, USA
}

\begin{abstract}
The Duke Free Electron Laser Laboratory is developing a booster synchrotron to provide full energy injection into the Duke electron storage ring. In this paper, we describe the development of the control system for the booster. Requirements include the competing needs of simple and reliable turn-key operation for the machine as a booster; and the sophistication and flexibility of operation of the machine as a storage ring for commissioning, machine studies and as a light source. To simplify operations and machine studies, the high level controls will present the system in terms of the physics quantities of the accelerator, allowing a tight integration between the physics model and the low level hardware control, as we have previously implemented for the Duke storage ring.
\end{abstract}

\section{INTRODUCTION}

The Duke booster synchrotron, currently in its installation phase, is designed to provide full energy injection into the existing Duke storage ring over an energy range from 0.27 to $1.2 \mathrm{GeV}$ [1]. This compact booster will allow single bunch extraction at any energy within this range to match the storage ring energy. Top-off mode will be supported to allow continuous operation of the high intensity gamma source associated with the storage ring free electron laser (FEL). Basic booster parameters are listed in Table 1. The control system will be built using the Experimental Physics and Industrial Control System (EPICS) [2] tool kit which has been in use at the Duke FEL Laboratory for accelerator and light source controls for more then ten years.

The booster's primary function is of course, that of an injector. As such, the control system needs to provide a simple, semi-autonomous means of coordinating the linear accelerator, injection of the electron bunches into the booster, ramping of electron energy within the booster, and extraction of the higher energy electron bunches into the storage ring.

Additionally, the control system will be developed in such a way as to allow operation similar to that of a storage ring. With limited additional diagnostics (some of which can be shared with the main ring) and other hardware, the booster can be configured to allow steady state operation and step-by-step adjustments in energy or other settings. This will be extremely useful during commissioning, in

\footnotetext{
* Work supported by U.S. Department of Energy grant DE-FG0201ER41175 and by U.S. AFOSR MFEL grant F49620-001-0370.

† hartman@fel.duke.edu
}

particular as a means of dealing with the high level of magnetic field saturation at the higher energies. Additionally, this allows the booster to have a secondary function as that of an electron storage ring. Since the main Duke storage ring does not require the continuous operation of the booster, the booster itself can be used independently. This compact ring can be a useful testbed for accelerator physics studies or even potentially developed to be an independent light source.

Table 1: Basic Parameters of the Duke Booster

\begin{tabular}{|l|r|}
\hline Injection energy & $0.27 \mathrm{GeV}$ \\
\hline Maximum beam energy & $1.2 \mathrm{GeV}$ \\
\hline Circumference & $31.902 \mathrm{~m}$ \\
\hline Bending radius & $2.273 \mathrm{~m}$ \\
\hline RF frequency & $178.547 \mathrm{MHz}$ \\
\hline Harmonic number & 19 \\
\hline Nominal operation cycle & $1.2-2.5$ seconds \\
\hline Energy rise time (minimal) & 0.55 seconds \\
\hline
\end{tabular}

\section{HIGH LEVEL CONTROLS}

The high level interface to the booster will be modeled on the approach used in the high level controls for the Duke storage ring. For the storage ring, the machine is presented to the operator or physicist in terms of the physics quantities of the accelerator [3]. For instance, rather then setting magnet power supplies in terms of current, the effective focusing strength of the magnets is controlled instead. This focusing strength, combined with the storage ring energy, is then mapped using the lower level software to control the appropriate power supply (or supplies) associated with that magnetic element. This mapping is done at a low level within the EPICS input/output controller (IOC) providing a simplified interface for the higher level of the operator interface. This approach simplifies storage ring setup and tuning, provides a coherent interface to multifunction magnets, and simplifies complex feed-forward schemes. Additionally, nonlinearities of the system, such as those due to saturation of magnetic elements, are dealt with at a lower level. This allows instead the linear operation of controlled physics quantities with the high level interface presenting a nearly energy independent machine. 


\section{Booster as a Dedicated Injector}

As a dedicated injector to the main storage ring, this approach simplifies machine setup. The user program of the Duke storage ring calls for frequent changes of machine setup, such as multiple ring energies over the course of a single operations shift. With the physics units to engineering units built in to the low level software, the ideal machine would require only one knob to set extraction energy. However, we will incorporate additional knobs for adjustments to tunes, chromaticity, orbit, etc. Each magnetic element will be described by an array indicating focusing strength, orbit offset, etc., as appropriate for that magnet, for each step of the ramp. An additional array common to all elements will indicate the energy. Low level software within the IOC will map the appropriate power supply settings based on the input of the relevant arrays, such as energy and orbit offset for an orbit corrector; or energy, focusing strength and tune adjustment for a quadrupole trim. The resulting array then provides the ramping waveform for the relevant power supply. Generating new waveforms will be accomplished by the normal tuning process used for setup. The resulting arrays can then be saved to snapshots and restored for later operations.

\section{Booster as a Storage Ring}

The functionality of the control system required to operate the booster as a storage ring is critical for initial commissioning. Essentially, it will make it possible to step through the arrays describing the magnetic elements of the ring, stopping at the appropriate index for any particular energy setting. When the arrays are no longer being clocked through, the behavior of the machine is that of a storage ring. By incorporating the ability to tune a section of the arrays around the index of the stopping point, it becomes possible to simultaneously tune the machine as if it were a storage ring, and adjust the ramping waveforms of a booster. The physics based approach to the high level interface allows this process to more closely mimic the physics models. Additionally, it simplifies such tasks a adjusting tunes or chromaticity which involve a number of magnetic elements since a single knob can be used to adjust the setting for a number of power supplies.

\section{LOW LEVEL CONTROLS}

The control system for the booster will make use of much of the same type of hardware as is currently in use for the main storage ring. This reduces additional device driver software development and simplifies operations and ongoing maintenance and support. Some areas requiring additional development are highlighted below.

\section{Ramping Power Supplies}

The Duke booster is designed such that its 12 dipole magnets, 8 focusing quadrupole magnets (of two types) and
8 defocusing quadrupole magnets are powered by a single power supply unit. The proper ratio of magnet strength of the quadrupoles is achieved by the type of coil and type of iron core. Additional ramping magnets include two sextupoles (focusing and defocusing), plus 12 dipole trims, 16 quadrupole trims, and 25 orbit trims including strong trims for an injection/extraction bump. Five different types of power supplies, all with analog external control, will power these magnets. VMEBus waveform generator boards (Joerger Enterprises model VDACM) will be used to provide the analog control signal. This board level hardware solution easily meets our requirements for speed, accuracy and reproducibility. It also offers greater flexibility in terms of setup and operation as compared to an analog circuit approach to ramping; and provides a simpler, uniform interface to a variety of power supplies as compared to digitally controlled power supplies.

\section{Injection and Extraction}

The injection and extraction will be an enhancement of the existing linac to storage ring injection system. A series of hardware and software triggers will control injection, ramping, and extraction [4]. This entire system will be under computer control, with triggers and appropriate delays for extraction produced and calculated within the IOC. The operation cycle of less then $1 \mathrm{~Hz}$ allows sufficient time to dynamically adjust kicker delays during the extraction period to sequentially extract individual booster bunches into selected ring buckets.

The control system will send an initial trigger pulse using a digital output module. This signal will then be synchronized to a $147 \mathrm{KHz}$ signal based on the $178.5 \mathrm{MHz}$ RF frequency and the harmonic numbers of 64 and 19 for the main storage ring and the booster, respectively. This signal will then be processed using VMEBus digital delay generators to set appropriate delays for injection for linac systems and booster injection kickers. The system will then generate another pulse which will be synchronized to the mains line frequency (for predictable impact from power supply ripple) and be used to begin the ramping of the booster magnets. The end of ramp from the waveform generators will produce an interrupt to begin the extraction process. Delays will be set to control extraction from any booster bunch to any storage ring bunch for the extraction kickers. This process will be such that it can run semiautonomously with the operator selecting the ring buckets available for extraction. Development of a reliable means of measuring charge per electron bunch will be required to support a more independent mode of operation for top-off mode.

\section{Machine Protection}

An area of concern for machine protection is overheating due to limitations in the existing water cooling system. Magnets will be equipped with thermal switches connected to the external interlock of the power supply units. This 
will provide the final level of machine protection. However, slower or long term changes in temperature are also of interest since they can be indicative of reduced cooling capacity for the system. The booster will employ approximately 100 low cost temperature sensors to monitor key components and the ambient area. The Duke storage ring is currently using over 400 digital thermometers (Dallas Semiconductor DS1820) using a "1-wire" network interfaced to the control system with an RS-232/ASCII to 1wire adaptor. These digital thermometers have been an asset in diagnosing such problems as reduced flow through the coils of magnets or improperly functioning flow valves. By monitoring archived data via EPICS, long term trends can be identified indicating areas of concern before they reach a level where a fault would interrupt operations. The booster will use the same or a similar sensor for this purpose. Although this is not a very robust technology, the relatively low cost per channel allows us to heavily instrument the machine with temperature sensors. In the event of a failure in this system, the thermal switches are in place to protect equipment

However, thermal switches are not appropriate for use in protecting the synchrotron absorbers from overheating in the event of a cooling system fault. These absorbers are incorporated into the arc vacuum chambers of the booster. Due to mechanical clearances, there is no room to incorporate a thermal switch. A failure in the cooling to these absorbers would be a critical problem. Any sensor must be located at the end of the $1170 \mathrm{~mm}$ dipole magnets and have sufficient resolution to detect a temperature rise through conduction. The digital thermometers do not have sufficient resolution for this, nor do they offer sufficient reliability or speed. For this purpose, we will use platinum resistance temperature detector (RTD) sensors. RTDs offer long term stability and repeatability with sufficient resolution over this range to identify any changes in cooling capacity of the absorbers over time.

\section{Diagnostics}

The booster will have diagnostic systems typical of a booster synchrotron. Nine sets of beam position monitors (BPMs) will be installed. Two stripline BPMs are designated for fast beam diagnostic measurements. The booster will have a beam current monitor. There will also be two fast beam current monitors: one on the linac-to-booster transfer line and one on the booster-to-ring transfer line. Instruments such as oscilloscopes and spectrum analyzers will be interfaced to the EPICS back end using the LinuxGPIB [5] software package and standard PC-based PCIGPIB hardware. This approach is currently in use for the Duke storage ring.

\section{CONCLUSION}

Presently, the majority of the required hardware for the control system has been purchased. All device driver support software has been written and tested. The higher level software is in development and should be ready prior to the beginning of the booster's commissioning.

\section{REFERENCES}

[1] S. F. Mikhailov, et al., "Status of the Booster Injector for the Duke FEL Storage Ring," in these proceedings.

[2] http://www.aps.anl.gov/epics/

[3] Y. K. Wu, S. Hartman, S. F. Mikhailov, "A Physics Based Control System for the Duke Storage Ring," PAC03, May 2003, Portland, Oregon.

[4] G. Kurkin, I. Pinaev, S. Hartman, S. Mikhailov, Y. Wu, "A New Timing System for the Duke Booster and Storage Ring," in these proceedings.

[5] http://linux-gpib.sourceforge.net/ 